\title{
Verso un 2015 pieno di progetti, idee e nuovi appuntamenti
}

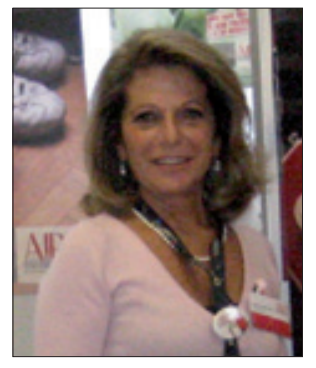

\author{
Luisa Sternfeld Pavia \\ Presidente \\ Associazione Italiana Rene \\ Policistico AIRP onlus \\ luisa.sternfeld.airp@renepolicistico.it
}

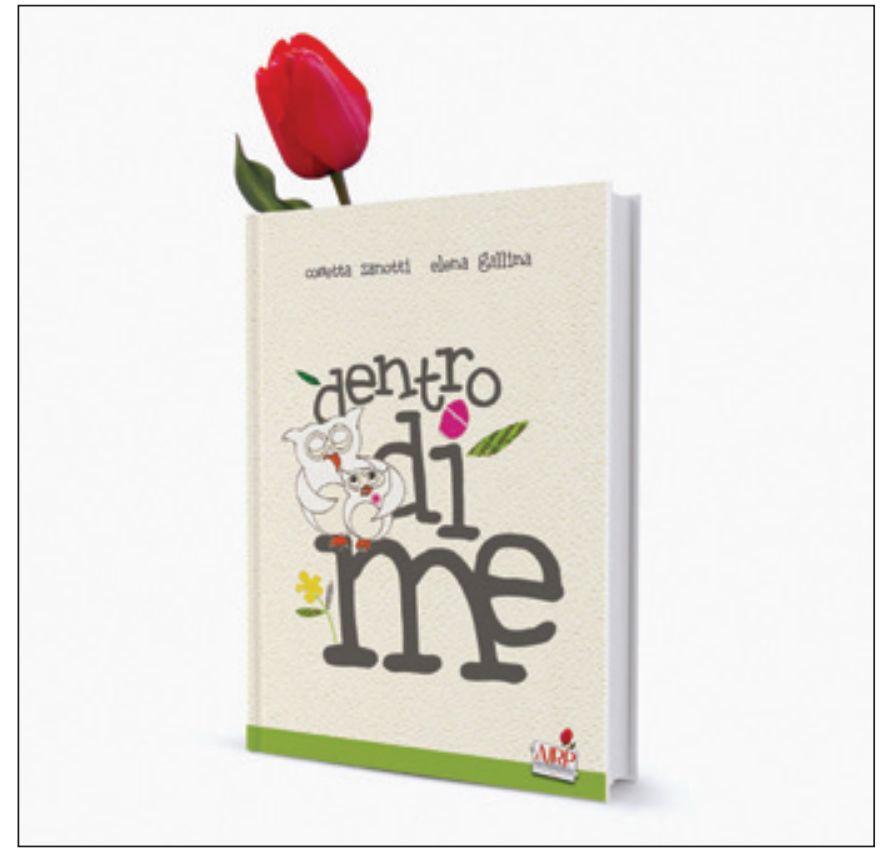

Fig. 1 - Il libro "Dentro di me".

Dall'organizzazione di corsi e seminari agli eventi locali, dalle campagne di comunicazione alla pubblicazione di libri, AIRP porta avanti senza sosta il proprio compito di supporto ai malati di rene policistico e di sostegno alla ricerca clinica, nella convinzione che sarà possibile in futuro rallentare o bloccare il decorso della patologia.

Siamo particolarmente fieri del fatto che incontri, tavole rotonde e simposi, organizzati da AIRP in tutta Italia, abbiano permesso a moltissimi pazienti e alle loro famiglie di acquisire informazioni importanti anche attraverso il contatto diretto con gli specialisti del settore.

L'ultimo trimestre dell'anno ci ha visto impegnati su diversi fronti.

A Roma, in collaborazione con l'Ospedale Bambino Gesù, abbiamo organizzato il primo Incontro Nazionale sulla forma recessiva del rene policistico, che ha riunito i massimi esperti nazionali e internazionali sull'argomento.

In quell'occasione, è stato presentato il libro Dentro di me (Fig. 1) di Cosetta Zanotti, illustrato da Elena Gallina. Un volume dedicato ai più piccoli, protagonistail gufetto Gugù. "Ognuno di noi ha dentro di sé l'arcobaleno", dice la storia, che parla con un linguaggio semplice e pieno di immaginazione. Perché ogni bambino, qualunque sia la sua condizione di salute, deve poter immaginare il proprio presente e il proprio futuro.

Questo libro è stato il primo gesto concreto che 1'Associazione ha rivolto ai più piccoli, con la volontà di dare a loro, e ai
Eccoci alla fine di un 2014 fitto di impegni e iniziative, come
Cari amici,

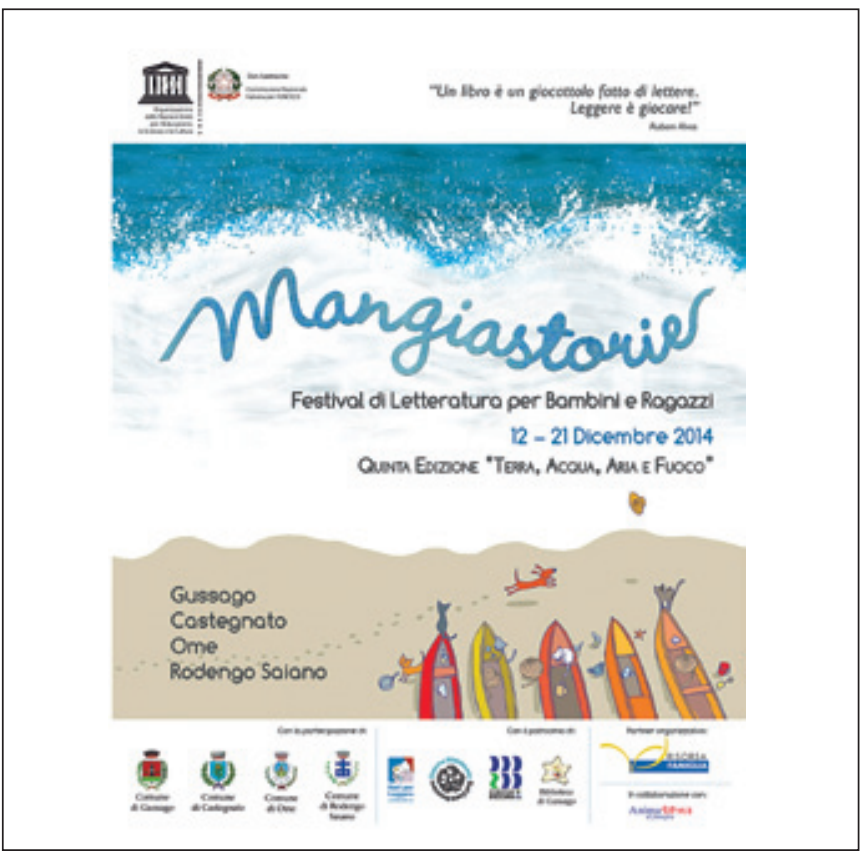

Fig. 2 - La locandina del Festival. 
loro familiari un forte e chiaro segnale di aiuto, affinché non si sentano né diversi né abbandonati. Il ricavato dell'iniziativa è completamente devoluto alla ricerca sulla malattia, che dal prossimo anno riguarderà anche la forma $\mathrm{ARPKD}$.

Il libro ha ricevuto una bellissima accoglienza ed è stato al centro di ulteriori iniziative, come la partecipazione di AIRP al Festival di letteratura per Bambini e Ragazzi "Mangiastorie" (Fig. 2), con un incontro che si è svolto a Gussago (Brescia) pochi giorni prima di Natale.

Con l'autrice, erano presenti il Professor Francesco Scolari, Primario di Nefrologia degli Spedali Civili di Brescia, sede di Montichiari, il Professor Luigi Paladin, esperto di letteratura per l'infanzia e Saverio Simonelli, responsabile dei programmi culturali Tv 2000.

Dentro di me è stato proposto anche a Desenzano del Garda, nel mese di dicembre, in un corner di raccolta fondi presso la locale Azienda Ospedaliera, e prima ancora a Milano, in occasione del $46^{\circ}$ Corso di aggiornamento in nefrologia e metodiche dialitiche della Fondazione D'Amico, un appuntamento di grande rilievo, con un programma scientifico molto corposo, al quale AIRP ha preso parte.

Non vi sarà sfuggita, inoltre, la campagna Pubblicità Progresso sul rene policistico, con lo spot andato in onda sulle reti RAI tra novembre e dicembre e sulle reti Mediaset a fine anno.

Cari amici, tutto ciò non sarebbe possibile senza il vostro concreto contributo. Portare avanti le attività in corso, organizzarne di nuove e soprattutto continuare a sostenere i progetti di ricerca: tutto questo ha bisogno della solidarietà e dell'aiuto di tutti i nostri soci, come è facile comprendere. Per questo vi invito a rinnovare la quota associativa, che per AIRP è assolutamente vitale. E vi dò appuntamento al nuovo anno con nuove idee, nuovi appuntamenti, nuova speranza.

Buon 2015 a tutti! 